\title{
Analysis of a three-component model phase diagram by catastrophe theory: Potentials with two order parameters
}

\author{
J. Gaite, ${ }^{*}$ J. Margalef-Roig, and S. Miret-Artés \\ Instituto de Matemáticas y Física Fundamental, Serrano 123, 28006 Madrid, Spain
}

(Received 23 July 1998; revised munuscript received 9 October 1998)

\begin{abstract}
In this work we classify the singularities obtained from the Gibbs potential of a lattice gas model with three components, two order parameters, and five control parameters applying the general theorems provided by catastrophe theory. In particular, we clearly establish the existence of Landau potentials in two variables or, in other words, corank-2 canonical forms that are associated with the hyperbolic umbilic, $D_{+4}$, its dual the elliptic umbilic, $D_{-4}$, and the parabolic umbilic $D_{5}$ catastrophes. The transversality of the potential with two order parameters is explicitly shown for each case. Thus we complete the catastrophe-theory analysis of the three-component lattice model, initiated in a previous paper [Phys. Rev. B 57, 13527 (1998)].
\end{abstract}

[S0163-1829(99)00713-4]

\section{INTRODUCTION}

The importance of phase transitions with several order parameters is very well known in different branches of physics. ${ }^{2}$ A great amount of theoretical work has been done in order to understand and construct accurate phase diagrams. As is well known, two different approaches are usually employed, one more phenomenological by introducing Landau polynomial potentials which try to describe experimental singular behaviors and the second one applying catastrophe theory (CT) (also known as singularity theory ${ }^{3-5}$ ) and thus adopting a more methodological point of view. Even in the second case there are several ways to deal with phase transitions and diagrams. Most works adopting the second point of view start with the canonical unfoldings as given and base their treatments on the effect of perturbations leading to preserving or not the internal symmetry of the system considered or, in other words, they focus their analysis in the symmetry-breaking character of some phase transitions. The procedure we adopt here is different since we begin with a thermodynamic potential (for example, the Gibbs potential), assuming a mean-field approach, and we apply an algorithm or program according to the general mathematical theorems established by CT, in order to extract all the topological information of the original thermodynamics potential. In a recent paper we have applied this CT program to a three-component model phase diagram and we have found for a one order-parameter potential the highest singularity with codimension five to be the wigwam or $A_{6}$ catastrophe. ${ }^{1}$

Our CT program could be very briefly stated as follows. ${ }^{1}$ Let $H(x, \pi)$ be a real function of state variables $x$ $=\left(x_{1}, \ldots, x_{n}\right)$ and control parameters $\pi=\left(\pi_{1}, \ldots, \pi_{r}\right)$. Then we must first pick a point $\left(x_{0}, \pi_{0}\right)$ such that $x_{0}$ is a degenerate critical point of $H$ and we consider the unfolding $h(x, \pi)=H\left(x+x_{0}, \pi+\pi_{0}\right)-H\left(x_{0}, \pi_{0}\right)$ and $g(x)=h(x, 0)$ to translate $x_{0}$ to the origin of coordinates. Second, we calculate the determinacy and codimension of $g$ from the $k$ jet of $g, j^{k}(g)$, and, third, we study the $k$ transversality of $h$. If this function is $k$ transversal we can affirm that $h$ and the canonical form of the unfolding of $g$ are isomorphic. Then we can replace the original $H$ function by this canonical unfolding. If not, we can state that the $H$ function is not susceptible to being studied by CT.

CT has not been usually applied in a rigorous way by taking into account all concepts and theorems needed for its correct implementation. The catastrophe program proposed here provides a very useful and systematic way to explore, examine and classify, with not very much computational effort, singularities and general behaviors of physical systems. In particular, we emphasize the study of transversality of the actual thermodynamical potentials which guarantees that those simple forms (polynomial potentials or canonical forms) represent indeed up to a diffeomorphism the original thermodynamical potential, this fundamental aspect being many times not properly considered. Thus some Landau (polynomial) potentials utilized to describe phase transitions in the literature may claim phenomenological value but may not claim to have a direct connection with the real thermodynamical potential, which is generally nonpolynomial, unless the CT analysis of the latter, including transversality, is performed.

Following our CT program we do not need to invoke any convention (for example, delay or Maxwell convention) in order to classify degenerate or nondegenerate critical points on the state variables space. Both conventions are not intrinsic to CT. Only when we deal with the time evolution or when dynamical considerations about the physical system are considered, could a given convention be advisable. In particular, when the order of a phase transition needs to be determined a convention is necessary because the transition occurs when an appropriate separatrix in the control parameter space is crossed.

Here we will focus our attention on the lattice-gas model for a system with three components which simulates, in particular, a binary fluid mixture. A wide literature has already been devoted to it from different points of view (Refs. 6-10, and references therein), restricted to the case with one order parameter. In fact, very few studies with these methods can be found for phase transitions with two order parameters. ${ }^{11}$ This is rather surprising since the Landau potential for the 
three-state Potts model, which is a particularly important three-component model, has long been known to have two order parameters. ${ }^{12} \mathrm{We}$ shall perform a complete CT study of the case with two order parameters, which is the maximum number for this model. The starting point will be the exact mean-field Gibbs potential.

This work is organized as follows: In Sec. II we describe the thermodynamical potential to be analyzed, give its physical interpretation, and apply the CT program to the potential previously introduced by considering the singularities with corank equal to 2 and establish the elementary catastrophes associated. In Sec. III, we analyze in more detail the Potts model as a particular case. The last section is devoted to a discussion of the previous results.

\section{ANALYSIS OF THE GIBBS POTENTIAL WITHIN THE FRAMEWORK OF CATASTROPHE THEORY}

In the mean-field theory, the Gibbs potential is a function of the concentration of two of the three components and depends on three thermodynamical parameters, which can be taken as the temperature and the chemical potentials of the two components, and on three molecular parameters. The phase diagram deduced from this function is an accurate description of the system, except close to the (multi)critical points, where fluctuations become important and alter significantly the mean-field theory predictions. For this reason, the Gibbs potential has been the basis for determining the overall phase diagram. ${ }^{9,10}$

Let us consider the reduced form of the Gibbs potential according to Ref. 9

$$
G(x, y, z, a, b, c)=a y z+b x z+c x y+x \ln x+y \ln y+z \ln z,
$$

where the parameters $a, b, c$ are related to some molecular interaction parameters; the variables $x, y, z$ are the mole fractions defined by $x=N_{x} / N, y=N_{y} / N$, and $z=N_{z} / N, N=N_{x}$ $+N_{y}+N_{z}$ being the number of total moles and $N_{x}, N_{y}$, and $N_{z}$ the moles of each component. The following constraint among the three variables is therefore required:

$$
x+y+z=1, \quad \text { with } \quad 0<x, y, z<1 .
$$

This potential has a wide range of applications, covering in particular the physics of binary fluid mixtures. ${ }^{13,14}$

From the constraint Eq. (2), we build a new function $H$ of two variables such that

$$
\begin{aligned}
H(x, y, a, b, c)= & a y(1-x-y)+b x(1-x-y)+c x y+x \ln x \\
& +y \ln y+(1-x-y) \ln (1-x-y) .
\end{aligned}
$$

The mean-field theory prescription is then to minimize the nonequilibrium Gibbs potential $H-\mu_{x} x-\mu_{y} y$,

$$
\begin{aligned}
\bar{H}\left(x, y, a, b, c, \mu_{x}, \mu_{y}\right)= & a y(1-x-y)+b x(1-x-y)+c x y \\
& +x \ln x+y \ln y+(1-x-y) \\
& \times \ln (1-x-y)-\mu_{x} x-\mu_{y} y
\end{aligned}
$$

with respect to $x$ and $y$, where $\mu_{x}$ and $\mu_{y}$ are related to differences between the chemical potentials of the three components. $^{9}$
CT will be applied to the $\bar{H}\left(x, y, a, b, c, \mu_{x}, \mu_{y}\right)$ function to classify the corank-2 singularities, at the generic point $\left(x_{0}, y_{0}\right)$ which moves on the triangle $x_{0}>0, y_{0}>0$ and 1 $-x_{0}-y_{0}>0$. CT conventionally uses the origin of coordinates as the point where singularities occur. Therefore, we shall translate the function $\bar{H}$ in order to have the singularities at the origin. This translated function is written now as

$$
\begin{aligned}
h\left(x, y, a, b, c, \mu_{x}, \mu_{y}\right)= & \bar{H}\left(x+x_{0}, y+y_{0}, a+a_{0}, b+b_{0}, c\right. \\
& \left.+c_{0}, \mu_{x}+\mu_{x, 0}, \mu_{y}+\mu_{y, 0}\right) \\
& -\bar{H}\left(x_{0}, y_{0}, a_{0}, b_{0}, c_{0}, \mu_{x, 0}, \mu_{y, 0}\right),
\end{aligned}
$$

and the germ of the unfolding $h$ is

$$
g(x, y)=h(x, y, 0,0,0,0,0)
$$

Now according to the CT program proposed in our previous paper $^{1}$ and mentioned in the Introduction, the following steps will be developed for our problem.

\section{A. Condition for a degenerate critical point at the origin}

The first step is to write the conditions for which the origin of coordinates $(0,0)$ is a degenerate critical point with corank equal to 2 of $g$. This can be done by equating all the first and second partial derivatives of $g$ to zero at the point $(0,0)$ (hypothesis $\left.\Sigma_{0}\right)$-thus the Hessian of the germ $g$ vanishes as well. This leads to five conditions among the variables and parameters $x_{0}, y_{0}, a_{0}, b_{0}, c_{0}, \mu_{x, 0}$, and $\mu_{y, 0}$. From that system of five equations the following relations can be extracted:

$$
\begin{gathered}
a_{0}=\frac{1}{2}\left(z_{0}^{-1}+y_{0}^{-1}\right), \quad b_{0}=\frac{1}{2}\left(z_{0}^{-1}+x_{0}^{-1}\right), \\
c_{0}=\frac{1}{2}\left(x_{0}^{-1}+y_{0}^{-1}\right),
\end{gathered}
$$

and

$$
\begin{aligned}
\mu_{x, 0}= & \frac{1}{2}\left(-y_{0} z_{0}^{-1}-x_{0} z_{0}^{-1}+z_{0} x_{0}^{-1}+y_{0} x_{0}^{-1}\right) \\
& +\ln x_{0}-\ln z_{0}, \\
\mu_{y, 0}= & \frac{1}{2}\left(-y_{0} z_{0}^{-1}-x_{0} z_{0}^{-1}+z_{0} y_{0}^{-1}+x_{0} y_{0}^{-1}\right) \\
& +\ln y_{0}-\ln z_{0},
\end{aligned}
$$

with the definition $z_{0}=1-x_{0}-y_{0}>0$. Equations (7) give rise to a surface with parameters $x_{0}$ and $y_{0}$ fulfilling the condition for a degenerate critical point with corank 2 at $(0,0)$.

\section{B. Classification of the germ $g$}

With the hypothesis $\Sigma_{0}$, the 3-jet of $g$ (Taylor expansion truncated beyond terms of degree 3 ) around the point $(0,0)$ is 


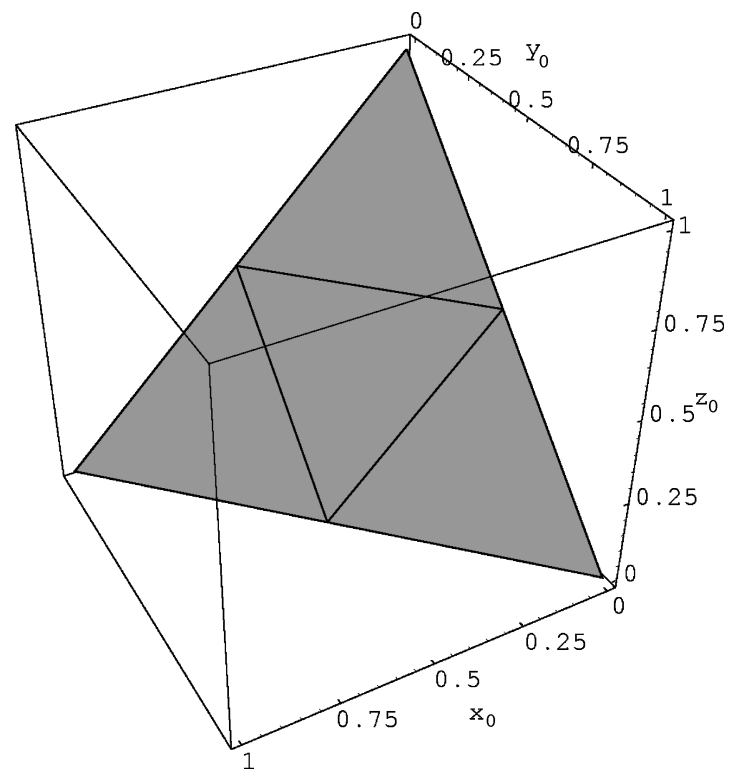

FIG. 1. Regions for $x^{3}-x y^{2}$ potentials (inner triangle) and for $x^{3}+x y^{2}$ potentials (three outer triangles) in $x_{0} y_{0} z_{0}$ space.

$$
\begin{aligned}
j^{3}(g)= & \frac{1}{6} x^{3}\left(-x_{0}^{-2}+z_{0}^{-2}\right)+\frac{1}{2} x^{2} y z_{0}^{-2}+\frac{1}{2} x y^{2} z_{0}^{-2} \\
& +\frac{1}{6} y^{3}\left(-y_{0}^{-2}+z_{0}^{-2}\right)
\end{aligned}
$$

This 3-jet can be considered now as an homogenous polynomial of degree equal to 3 and can be rewritten as

$$
j^{3}(g)=a_{1} x^{3}+a_{2} x^{2} y+a_{3} x y^{2}+a_{4} y^{3},
$$

with $a_{1}=(1 / 6)\left(-x_{0}^{-2}+z_{0}^{-2}\right), a_{2}=a_{3}=(1 / 2) z_{0}^{-2}$ and $a_{4}$ $=(1 / 6)\left(-y_{0}^{-2}+z_{0}^{-2}\right)$. We know from singularity theory that a general non-null homogeneous polynomial of degree 3 is equivalent by a linear transformation to one and only one of the following germs: $x^{3}-x y^{2}, x^{3}+x y^{2}, x^{2} y$ and $x^{3} .15,16$ We must remark that these $x$ and $y$ are not to be identified with the initial physical variables, though they are linearly related to them. The application of this lemma to Eq. (10) to classify the 3 -jet is given in Appendix A and here only the final conclusions will be summarized:

(1) If $\left(1-2 x_{0}\right)\left(1-2 y_{0}\right)\left(1-2 z_{0}\right)=0$ then $j^{3}(g) \sim x^{2} y$,

(2) If $\left(1-2 x_{0}\right)\left(1-2 y_{0}\right)\left(1-2 z_{0}\right)>0$ then $j^{3}(g) \sim x^{3}$ $+x y^{2}$

(3) If $\left(1-2 x_{0}\right)\left(1-2 y_{0}\right)\left(1-2 z_{0}\right)<0$ then $j^{3}(g) \sim x^{3}$ $-x y^{2}$.

All of these three cases can be collected in a plot shown in Fig. 1. In Fig. 1 we display in the $x_{0} y_{0} z_{0}$ space the regions for the $x^{3} \pm x y^{2}$ germs and in Fig. 2 the separatrices between regions with different codimensions in parameter space. The separatrix $x_{0}=1 / 2$, say, in conjuction with the equations defining the instability surface (7) gives rise to the plane $-a_{0}$ $+b_{0}+c_{0}=2$, used in Fig. 2 to find the separatrix in parameter space, which is a hyperbola branch.

The equivalence between the 3 -jet and the canonical germs implies that their codimensions are equal. Thus we have that

$$
\operatorname{cod}\left(j^{3}(g)\right)=\operatorname{cod}\left(x^{2} y\right)=\infty,
$$

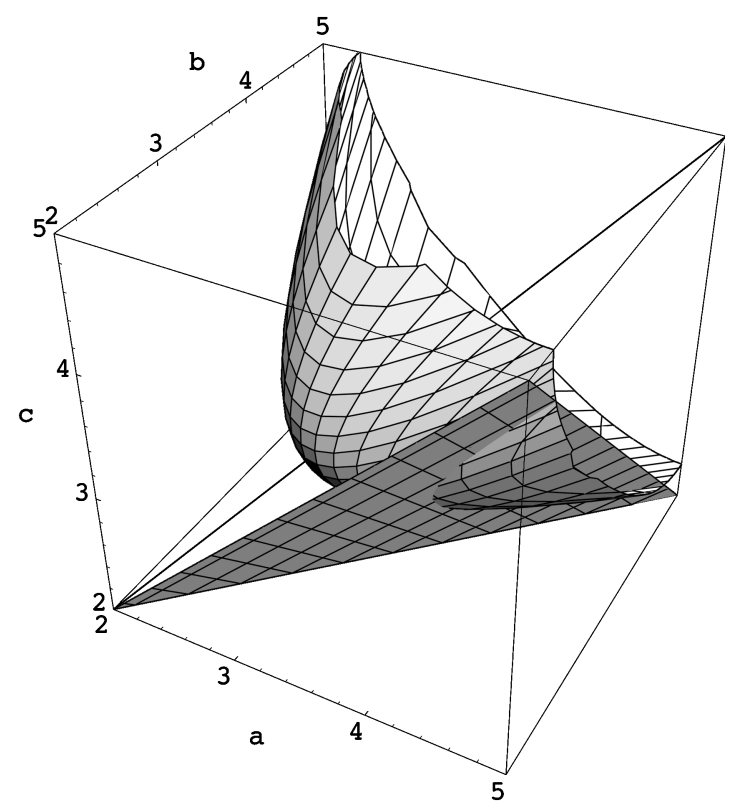

FIG. 2. Phase diagram showing the corank-2 instability surface. The intersection of the plane and the surface gives one of the three branches of the boundary between the $x^{3}+x y^{2}$ and $x^{3}-x y^{2}$ potentials. The diagonal corresponds to the three-state Potts model. (See text).

$$
\begin{aligned}
& \operatorname{cod}\left(j^{3}(g)\right)=\operatorname{cod}\left(x^{3}-x y^{2}\right)=3, \\
& \operatorname{cod}\left(j^{3}(g)\right)=\operatorname{cod}\left(x^{3}+x y^{2}\right)=3,
\end{aligned}
$$

and we observe that the behavior of the codimension of the 3 -jet is discontinuous according to Fig. 1. Indeed, when the point $\left(x_{0}, y_{0}\right)$ crosses the inner triangle the codimension of the 3-jet jumps to infinity. The codimension is only finite when what we could call hypothesis $\Sigma_{1}$ is fulfilled, that is,

$$
\Sigma_{1} \equiv\left(1-2 x_{0}\right)\left(1-2 y_{0}\right)\left(1-2 z_{0}\right) \neq 0 .
$$

On the contrary, if $\Sigma_{1}=0$, corresponding to the sides of the inner triangle, the germ $g$ is not 3-determinate and we have to increase the order of its jet by one more degree to explore 4-determinacy. We have that

$$
\begin{aligned}
j^{4}(g)= & a_{1} x^{3}+a_{2} x^{2} y+a_{3} x y^{2}+a_{4} y^{3}+b_{1} x^{4}+b_{2} x^{3} y \\
& +b_{3} x^{2} y^{2}+b_{4} x y^{3}+b_{5} y^{4}
\end{aligned}
$$

where now the $b$ coefficients are found to be

$$
\begin{gathered}
b_{1}=\left(x_{0}^{-3}+z_{0}^{-3}\right) / 12, \quad b_{2}=z_{0}^{-3} / 3, \quad b_{3}=z_{0}^{-3} / 2, \\
b_{4}=z_{0}^{-3} / 3, \quad b_{5}=\left(y_{0}^{-3}+z_{0}^{-3}\right) / 12 .
\end{gathered}
$$

As before, in order to classify the 4-jet of $g$ we invoke again a lemma of the singularity theory (see Appendix A). In all of the subcases examined the 4 -jet is equivalent to the canonical germ $x^{2} y+y^{4}$, it is 4-determinate and its codimension is equal to 4 .

\section{Determinacy and codimension of $g(x, y)$}

Once we have shown that the 3 -jet is equivalent to the canonical germs $x^{3} \pm x y^{2}$, and the 4-jet to $x^{2} y+y^{4}$, it is clear 
that as these germs are 3- and 4-determinate, the 3-jet and 4-jet will be also 3- and 4-determinate, respectively. Then $g \sim x^{3}-x y^{2} \quad\left(\right.$ for $\left.\Sigma_{1}<0\right), g \sim x^{3}+x y^{2} \quad\left(\right.$ for $\Sigma_{1}>0$ ) both with $\operatorname{cod}(g)=3$, and $g \sim x^{2} y+y^{4}$ (for $\Sigma_{1}=0$ ) but with $\operatorname{cod}(g)=4$. These equivalences exist up to an unknown change of coordinates, so that here and in the following the variables $x$ and $y$ have no physical interpretation and may be regarded as dummy variables.

\section{Canonical unfoldings of the germs $x^{3} \pm x y^{2}$ and $x^{2} y+y^{4}$}

It is well known from CT that $\left\{[x],[y],\left[x^{2}\right]\right\}$ is a basis for the quotient vector spaces $\langle x, y\rangle / \Delta\left(x^{3}-x y^{2}\right)$ and $\langle x, y\rangle / \Delta\left(x^{3}+x y^{2}\right) \quad$ and $\quad\left\{[x],[y],\left[x^{2}\right],\left[y^{2}\right]\right\}$ for $\langle x, y\rangle / \Delta\left(x^{2} y+y^{4}\right)$. Moreover, the $k$ transversal (for all $k$ $>0$ ) canonical unfolding of the canonical form $x^{3}-x y^{2}(e l-$ liptic umbilic, $D_{-4}$ ) and its dual $x^{3}+x y^{2}$ (hyperbolic umbilic, $D_{+4}$ ) and $x^{2} y+y^{4}$ (parabolic umbilic, $D_{5}$ ), are, respectively,

$$
\begin{aligned}
& x^{3}-x y^{2}+\lambda_{1} x+\lambda_{2} y+\lambda_{3} x^{2}, \\
& x^{3}+x y^{2}+\lambda_{1} x+\lambda_{2} y+\lambda_{3} x^{2},
\end{aligned}
$$

and

$$
x^{2} y+y^{4}+\lambda_{1} x+\lambda_{2} y+\lambda_{3} x^{2}+\lambda_{4} y^{2} .
$$

The corresponding bifurcation diagrams are well known in the singularity theory ${ }^{17}$ and can be seen in any of the standard books on this theory. ${ }^{4}$ Equations governing such bifurcation diagrams are

$$
\begin{aligned}
B_{D_{-4}}: 3 x^{2}-y^{2}+\lambda_{1}+2 \lambda_{3} x=0, & -2 x y+\lambda_{2}=0, \\
3 x^{2}+x \lambda_{3}+y^{2} & =0, \\
B_{D_{+4}}: 3 x^{2}+y^{2}+\lambda_{1}+2 \lambda_{3} x & =0, \quad 2 x y+\lambda_{2}=0, \\
3 x^{2}+x \lambda_{3}-y^{2} & =0,
\end{aligned}
$$

and

$$
\begin{gathered}
B_{D_{5}}: 2 x y+\lambda_{1}+2 \lambda_{3} x=0, \quad x^{2}+4 y^{3}+\lambda_{2}+2 \lambda_{4} y=0, \\
6 y^{3}+y \lambda_{4}+6 \lambda_{3} y^{2}-x^{2}+\lambda_{3} \lambda_{4}=0,
\end{gathered}
$$

which are obtained by equating to zero the first derivatives and Hessian of $g$ in each case.

\section{E. Canonical five unfolding of $g$}

Since $h$ has five parameters, the preceding unfoldings have to be extended with two or one irrelevant parameters in order to apply the isomorphy theorem. Thus, for example, for the canonical unfoldings $x^{3} \pm x y^{2}$, the two new unfoldings denoted by $\beta_{1}$ and $\beta_{2}$ can be written as

$$
\beta_{1}\left(x, y, \lambda_{1}, \ldots, \lambda_{5}\right)=x^{3}-x y^{2}+\lambda_{1} x+\lambda_{2} y+\lambda_{3} x^{2}
$$

and

$$
\beta_{2}\left(x, y, \lambda_{1}, \ldots, \lambda_{5}\right)=x^{3}+x y^{2}+\lambda_{1} x+\lambda_{2} y+\lambda_{3} x^{2}
$$

and they are $k$-transversal unfoldings for all $k>0$ of $x^{3}$ $-x y^{2}$ and $x^{3}+x y^{2}$, respectively. Now the bifurcation sets are $B_{\beta_{1}}=B_{D_{-4}} \times \mathbb{R}^{2}$ and $B_{\beta_{2}}=B_{D_{+4}} \times \mathbb{R}^{2}$.

In both cases, we can affirm that there is a change of coordinates (a diffeomorphism) $\varphi_{\left(x_{0}, y_{0}\right)}$ such that $g=\left(x^{3}\right.$ $\left.-x y^{2}\right) \cdot \varphi_{\left(x_{0}, y_{0}\right)}$ and the same holds for $x^{3}+x y^{2}$. Consequently,

$$
\bar{\beta}_{1}\left(x, y, \lambda_{1}, \cdots, \lambda_{5}\right)=s^{3}-s t^{2}+\lambda_{1} s+\lambda_{2} t+\lambda_{3} s^{2}
$$

and

$$
\bar{\beta}_{2}\left(x, y, \lambda_{1}, \ldots, \lambda_{5}\right)=s^{3}+s t^{2}+\lambda_{1} s+\lambda_{2} t+\lambda_{3} s^{2},
$$

where $(s, t)=\varphi_{\left(x_{0}, y_{0}\right)}(x, y)$, is a three-tranversal unfolding of $g$ with five parameters. Moreover, for the bifurcation sets we have that $B_{\bar{\beta}_{1}}=B_{\beta_{1}}$ and $B_{\bar{\beta}_{2}}=B_{\beta_{2}}$. A similar reasoning can be used for the canonical unfolding $x^{2} y+y^{4}+\lambda_{1} x+\lambda_{2} y$ $+\lambda_{3} x^{2}+\lambda_{4} y^{2}$ but now we have only one irrelevant parameter.

Finally, these canonical unfoldings of $g$ need to be related to the function $h$ or translated Gibbs potential. This is shown explicitly in next subsection through the so-called transversality condition.

\section{F. $k$ transversality of the translated function $h$}

Now we return to the translated Gibbs potential $h$ to establish its relation with the unfolding of $g$ studied above. The condition for the existence of this relation is its transversality. Since we will be dealing with the physical function $h$ its arguments will be the original physical variables, to be distiguished from the dummy variables used before. The vector space of the transversality, $V_{h}$, is defined by the first partial derivatives of $h$ with respect to the five parameters according to

$$
\begin{gathered}
V_{h}=\left\langle D_{a} h(x, y, 0,0,0,0,0)-D_{a} h(0,0,0,0,0,0,0),\right. \\
D_{b} h(x, y, 0,0,0,0,0)-D_{b} h(0,0,0,0,0,0,0), \\
D_{c} h(x, y, 0,0,0,0,0)-D_{c} h(0,0,0,0,0,0,0), \\
D_{\mu_{x}} h(x, y, 0,0,0,0,0)-D_{\mu_{x}} h(0,0,0,0,0,0,0), \\
\left.D_{\mu_{y}} h(x, y, 0,0,0,0,0)-D_{\mu_{y}} h(0,0,0,0,0,0,0)\right\rangle_{\mathrm{R}},
\end{gathered}
$$

where $\langle\cdots\rangle_{\mathbb{R}}$ means all the linear combinations with real coefficients.

Let us analyze the transversality of the unfolding $h\left(x, y, a, b, c, \mu_{x}, \mu_{y}\right)$. As was mentioned in the Introduction, this study is carried out in order to show that the function $h$ and the canonical unfolding of the germ $g$ are isomorphic. The vector space of the transversality, $V_{h}$, is defined by Eq. (18) and reads in our case

$$
\begin{aligned}
V_{h}= & \left\langle-y x-y^{2}+y z_{0}-y_{0} x-y_{0} y,-x^{2}-x y+x z_{0}-x_{0} x\right. \\
& \left.-x_{0} y, x y+x y_{0}+x_{0} y,-x,-y\right\rangle_{\mathbb{R}} .
\end{aligned}
$$

Our next step is to prove that $h$ is three or four transversal, according to each case. For this goal, we invoke one of the 
main theorems on $k$ transversality which states that $h$ will be $k$ transversal when the following algebraic condition is met: ${ }^{15,16}$

$$
\langle x, y\rangle=\Delta(g)+V_{h}+\langle x, y\rangle^{k+1},
$$

which is fulfilled in our case for $k=3$ or 4 . This requirement is proved in Appendix $B$ with the hypothesis $\Sigma_{0}$ and $\Sigma_{1}$ $\neq 0$ or $\Sigma_{1}=0$, respectively. We finally conclude that $h$ is a three- or four-transversal unfolding of $g$ with five parameters. Moreover, by using the main theorem on $k$ transversality, and for the three-transversal case, $\bar{\beta}_{1}$ and $\bar{\beta}_{2}$ are isomorphic to $h$, that is, there are three diffeomorphisms and a perturbation of parameters depending on $\left(x_{0}, y_{0}\right)$ for each case such that

$$
\begin{aligned}
h\left(x, y, a, b, c, \mu_{x}, \mu_{y}\right)= & \bar{H}\left(x+x_{0}, y+y_{0}, a+a_{0}, b+b_{0}, c\right. \\
& \left.+c_{0}, \mu_{x}+\mu_{x, 0}, \mu_{y}+\mu_{y, 0}\right) \\
& -\bar{H}\left(x_{0}, y_{0}, a_{0}, b_{0}, c_{0}, \mu_{x, 0}, \mu_{y, 0}\right) \\
= & s^{3} \pm s t^{2}+\lambda_{1} s+\lambda_{2} t+\lambda_{3} s^{2} \\
& +\varepsilon_{\left(x_{0}, y_{0}\right)}\left(a, b, c, \mu_{x}, \mu_{y}\right),
\end{aligned}
$$

where the diffeomorphisms take the following expressions:

$$
\begin{aligned}
\psi_{\left(x_{0}, y_{0}\right)}\left(x, y, a, b, c, \mu_{x}, \mu_{y}\right) & =\left(u, v, \lambda_{1}, \ldots, \lambda_{5}\right) \\
\varphi_{\left(x_{0}, y_{0}\right)}(u, v) & =(s, t) \\
\eta_{\left(x_{0}, y_{0}\right)}\left(a, b, c, \mu_{x}, \mu_{y}\right) & =\left(\lambda_{1}, \ldots, \lambda_{5}\right)
\end{aligned}
$$

with

$$
\psi_{\left(x_{0}, y_{0}\right)}(x, y, 0,0,0,0,0)=(x, y, 0,0,0,0,0) .
$$

All of these diffeomorphisms preserve the origin of coordinates. Moreover, concerning the bifurcations, we have that $\eta_{\left(x_{0}, y_{0}\right)}\left(B_{h}\right)=B_{D_{-4}} \times \mathbb{R}^{2}$ or $\eta_{\left(x_{0}, y_{0}\right)}\left(B_{h}\right)=B_{D_{+4}} \times \mathrm{R}^{2}$. Similar expressions can be written for the four-transversal case.

\section{THE THREE-STATE POTTS MODEL}

The natural (and oldest) generalization of the Ising model consists of taking a site variable which can take three equivalent states instead of two, constituting the three-state Potts model. (Similarly, one can define the $q$-state Potts model.) It has complete permutation symmetry among the three states, yielding a Gibbs potential corresponding to Eq. (1) with $a$ $=b=c$, Fig. 2, leaving as the only parameter the temperature $T$. The corank- 2 instability occurs for $T_{c}=1 / 9 \simeq 0.1111$ and the corresponding potential is the $D_{-4}$ germ. The threeparameter unfolding Eq. (14) contains the possible perturbations of temperature or chemical potentials. Since in the three-state Potts model only the temperature perturbation is allowed we must have a one-parameter unfolding. Its form is best deduced by symmetry arguments. ${ }^{12}$ We can substitute the germ by the symmetric form

$$
z^{3}+\bar{z}^{3}=x^{3}-3 x y^{2}
$$

where we have introduced the complex variable $z=x+i y$. The permutation symmetry is obviously generated by the discrete rotations $z \rightarrow e^{i(\pi / 3)} z$ and the reflection $z \rightarrow \bar{z}$. The temperature perturbation preserves the symmetry and belongs to the vector space $\langle x, y\rangle / \Delta\left(j^{3}(g)\right)$. Hence, it must be $x^{2}+y^{2}=z \bar{z}$. The corresponding unfolding is

$$
z^{3}+\bar{z}^{3}+\lambda z \bar{z}=x^{3}-3 x y^{2}+\lambda\left(x^{2}+y^{2}\right),
$$

where $\lambda$ is a monotonic function of the temperature, at least, in a neighborhood of $T_{c}$.

Now there arises the problem that the unfolding Eq. (25), being a section of the complete unfolding of $D_{-4}$, contains potentials with one minimum and three saddle points if $\lambda$ $>0$ or with one maximum and three saddle points if $\lambda<0$. These potentials are not bounded below nor have the three minima to be expected in this model. We must recall here the local character of CT and the discussion in Ref. 1. There we remarked that the global character of these potentials can only be established by a numerical study over the entire range of the variables $x$ and $y$. Thus one finds that they indeed have three minima, far from the point $(0,0)$ and distributed symmetrically, if we keep the temperature in a neighborhood of $T_{c}$. For a large value of $T$ only the minimum at $(0,0)$ survives. This is physically sensible, for at high temperature only the symmetric disordered phase can remain. The temperature $T_{c}$ precisely signals the point at which the symmetric disordered phase becomes unstable and disappears. Potentials for various situations are plotted in Fig. 3.

Note that for $T>T_{c}$ the three minima converge to $(0,0)$, eventually merging there. Since their depth decreases as the depth of the minimum at $(0,0)$ increases, there must be a value of $T$ such that they are equal. If we adopt the Maxwell convention then we have a first-order phase transition. This phase transition is known to occur in this model in space dimension $d=4$ and almost certainly for $d \geqslant 3 .{ }^{18}$ In $d=2$ the phase transition is definitely of second order, ${ }^{12}$ which means that the mean-field theory potential with which we started is insufficient in low dimensions to describe accurately the real character of the possible phase transitions.

Landau potentials for the three-state Potts model that are bounded below have appeared in the literature. ${ }^{19,20,12}$ They are commonly derived by renormalization-group arguments. In particular, it has been shown that for $d>3$ the only RG relevant term further from the cubic is the quartic symmetric term $\left(x^{2}+y^{2}\right)^{2}=(z \bar{z})^{2}$. It is even possible to prove with the powerful methods of $2 d$ conformal field theory that this term, in addition to the cubic term, constitute a well-defined Landau potential. ${ }^{12}$ So the potential $c\left(z^{3}+\bar{z}^{3}\right)+(z \bar{z})^{2}$ seems to be adequate for this model. In fact, due to the addition of the quartic term, it exhibits the same behavior as the total nonpolynomial potential displayed in Fig. 3. In any event, we must remark that the quartic term does not belong to the germ, as calculated before. However, it could well arise from a more refined Gibbs potential, namely, of the type obtained with cluster variation methods, ${ }^{21}$ especially if symmetry arguments are invoked like in Ref. 12. 

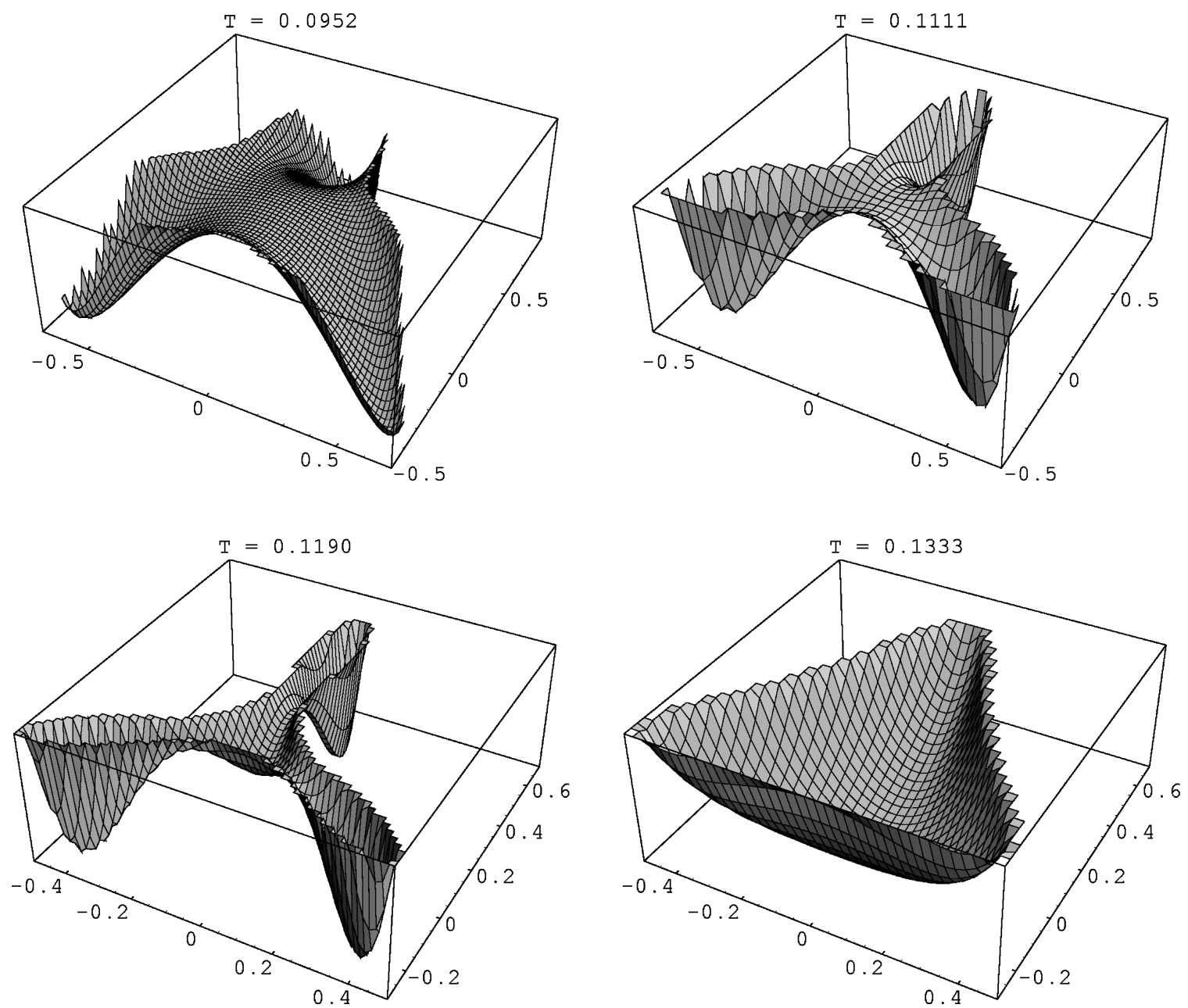

FIG. 3. Potentials for the three-state Potts model at various temperatures.

\section{CONCLUSION}

We have analyzed the possible modes of instability and further singularities of the mean-field theory Gibbs potential for a three-component model in the case that two order parameters are needed for their description. Since the case of one order parameter has been already studied in Ref. 1, the study of this Gibbs potential is now complete. We have found that three new catastrophes take place, namely, the hyperbolic umbilic, $D_{+4}$, its dual the elliptic umbilic, $D_{-4}$, and the parabolic umbilic, $D_{5}$, catastrophes. The parabolic umbilic has codimension 4 , which is the highest we can reach in the case of two control parameters, unlike the case of one control parameter studied in Ref. 1 where codimension five was reached. The germ and unfolding of the elliptic umbilic catastrophe are precisely (isomorphic to) those of the three-state Potts model, which is the model with the highest symmetry, and generally show three and even four phase coexistence. The total potential for the models belonging to the hyperbolic umbilic class do not have more than one local minimum and therefore they cannot give rise to phase coexistence. The phase structure for the parabolic umbilic is fairly complicated and can be seen in any of the standard books about CT. ${ }^{4,17}$ Finally, it is remarkable that with two order parameters the highest codimension 5, which should give rise to even more complex singularities, is not reached.
We must say a few words about natural generalizations. Instead of limiting ourselves to three components we could well envisage the case of $q$ components. This would include most models ever considered in the physics of phase transitions. The most symmetric case is the $q$-state Potts model. An essential advantage of increasing $q$ is that mean-field theory becomes more accurate-in fact becoming exact for space dimension $d \geqslant 2$ if $q \geqslant 4 .{ }^{18}$ A Landau potential for the $q$-state Potts model with $q-1$ order parameters is already known. ${ }^{20}$ Presumably, the rigorous study of the highest corank instabilities of the $q$ component Gibbs potential would produce a germ equal to the cubic term of that Landau potential, as for the three-state Potts model. The corresponding singularities exhibit the interesting feature of having modular parameters ${ }^{22}$ already for $q=4$. There would certainly be a flock of other singularities with the same corank and the same or higher codimension, and with lower corank. We leave to the entrepreneurial reader the exploration of this endless world of mathematical entities and their physical application.

\section{ACKNOWLEDGMENTS}

This work has been supported by DGICYT-Spain with Grant Nos. PB96-0887, PB96-0651-C03-01, and PB95-0071. 


\section{APPENDIX A}

The application of the lemma of CT to Eq. (10) to classify the 3 -jet can be carried out in a systematic way considering the following subcases: $(A)$ if $a_{4} \neq 0$ (that is, $2 y_{0} \neq 1-x_{0}$ ), then the auxiliary cubic equation of Eq. (10), obtained by dehomogenizing the polynomial, is given by

$$
0=\frac{a_{1}}{a_{4}}+\frac{a_{2}}{a_{4}} t+\frac{a_{3}}{a_{4}} t^{2}+t^{3} .
$$

Its discriminant is defined as $\Delta_{1}^{2}=\left(t_{1}-t_{2}\right)^{2}\left(t_{2}-t_{3}\right)^{2}\left(t_{3}\right.$ $\left.-t_{1}\right)^{2}$, where $t_{1}, t_{2}$, and $t_{3}$ are the roots of the cubic equation (A1). It can be expressed as

$$
\begin{aligned}
\Delta_{1}^{2} & =-4 p^{3} r-27 r^{2}+18 p q r-4 q^{3}+p^{2} q^{2} \\
& =\frac{1}{48 a_{4}^{4}} x_{0}^{-4} y_{0}^{-4} z_{0}^{-4}\left(1-2 x_{0}\right)\left(1-2 y_{0}\right)\left(1-2 z_{0}\right)
\end{aligned}
$$

with the following definitions: $p=-a_{3} / a_{4}, q=a_{2} / a_{4}$ and $r=-a_{1} / a_{4}$. Notice the following subcases:

$\left(A_{1}\right)$ Eq. (A1) has three equal real roots $\left(\Delta_{1}^{2}=0\right)$; this subcase is not possible because $y_{0}>0$,

$\left(A_{2}\right)$ Eq. (A1) has three real roots but two of them are equal (that is, $\left.\Delta_{1}^{2}=0\right)$; in this subcase, $j^{3}(g)$ and the monomial $x^{2} y$ are equivalent or, in mathematical terms, $j^{3}(g)$ $\sim x^{2} y$ (that is, the 3 -jet and the monomial are equal up to a change of coordinates which essentially implies that $a_{4}$ $\neq 0)$,

$\left(A_{3}\right)$ Eq. (A1) has three distinct real roots (that is, $\Delta_{1}^{2}$ $>0)$ and $j^{3}(g) \sim x^{3}-x y^{2}$, and

$\left(A_{4}\right) \mathrm{Eq}$. (A1) has two conjugate complex roots (that is, $\left.\Delta_{1}^{2}<0\right)$ and then $j^{3}(g) \sim x^{3}+x y^{2}$,

(B) if $a_{4}=0$ and $a_{1} \neq 0$ (that is, $2 y_{0}=1-x_{0}$ and $2 x_{0} \neq 1$ $-y_{0}$ ), then Eq. (A1) is replaced now by the auxiliary equation

$$
0=\frac{a_{3}}{a_{1}} t+\frac{a_{2}}{a_{1}} t^{2}+t^{3}
$$

and its discriminant $\Delta_{2}^{2}$ is written as

$$
\Delta_{2}^{2}=-4 q^{3}+p^{2} q^{2}=K\left(x_{0}, y_{0}\right)\left(2-3 x_{0}-2 y_{0}\right)
$$

with $K\left(x_{0}, y_{0}\right)>0$. The following redefinitions are now used: $p=-a_{2} / a_{1}, q=a_{3} / a_{1} ; t_{1}, t_{2}$ and $t_{3}$ are again the new roots of the cubic equation, Eq. (A3). Thus we have again the following subcases:

$\left(B_{1}\right)$ Eq. (A3) has three equal real roots. This is not possible since $a_{2}=a_{3} \neq 0$,

$\left(B_{2}\right)$ Eq. (A3) has three real roots, two of them equal $\left(\Delta_{2}^{2}=0\right)$; this condition implies that $3 x_{0}+2 y_{0}=2$ and then $j^{3}(g) \sim x^{2} y$,

$\left(B_{3}\right)$ Eq. (A3) has three distinct real roots $\left(\Delta_{2}^{2}>0\right)$, then $2>3 x_{0}+2 y_{0}$ and $j^{3}(g) \sim x^{3}-x y^{2}$, and

$\left(B_{4}\right)$ Eq. (A3) has two conjugate complex roots $\left(\Delta_{2}^{2}\right.$ $<0)$, then $2<3 x_{0}+2 y_{0}$ and $j^{3}(g) \sim x^{3}+x y^{2}$,

(C) Finally we have the case where $a_{4}=a_{1}=0$ (or $x_{0}$ $\left.=y_{0}=1 / 3\right)$ and we obtain that $j^{3}(g) \sim x^{3}-x y^{2}$. Due to the fact this case is related to the well-known Potts model, a more detailed analysis of this case will be addressed in Sec. III.

For the 4-jet $\left(\Sigma_{1}=0\right)$, the following subcases can be considered:

$\left(D_{1}\right)$ if $a_{4} \neq 0$ (that is, $2 y_{0} \neq 1-x_{0}$ ), then we take the auxiliary cubic equation

$$
0=a_{1}+a_{2} t+a_{3} t^{2}+a_{4} t^{3} .
$$

Equation (A5) can have only three real roots of which two are equal. With the linear transformation

$$
\psi^{-1} \equiv u=-a_{4} t_{1} x+a_{4} y, \quad v=-t_{2} x+y
$$

with $t_{1}$ the single root and $t_{2}$ the double root of Eq. (A5), then

$$
j^{3}(g) \psi(u, v)=u v^{2}
$$

The value for the double root $t_{2}$ is easily obtained from Eq. (A5) and its derivative with respect to the variable $t$ since both equations are satisfied for $t_{2}$ :

$$
\begin{aligned}
t_{2} & =\frac{a_{2} a_{3}-9 a_{1} a_{4}}{2\left(3 a_{2} a_{4}-a_{3}^{2}\right)}=\frac{1+2 x_{0}\left(-1+y_{0}\right)-2 y_{0}}{2 x_{0}^{2}} \\
& = \begin{cases}-2 y_{0} & \text { if } x_{0}=1 / 2, \\
\frac{-1}{2 x_{0}} & \text { if } y_{0}=1 / 2, \\
-1+\frac{1}{2 x_{0}} & \text { if } z_{0}=1 / 2 .\end{cases}
\end{aligned}
$$

One can further obtain $t_{1}$ and it coincides with $t_{2}$ only on the vertices of the triangle, where a concentration vanishes. Therefore, this situation, which would lead to a different singularity with 3 -jet $u^{3}$, is actually outside the domain we consider.

We can write the 4-jet in the new variables as

$j^{4}(g) \psi(u, v)=u v^{2}+c_{1} u^{4}+c_{2} u^{3} v+c_{3} u^{2} v^{2}+c_{4} u v^{3}+c_{5} v^{4}$,

where the new coefficients $c_{i}$ are linear combinations of the $b_{i}$. Only the sign of the nonzero $c_{1}$ coefficient is needed since, according to the mentioned lemma, the 4 -jet is equivalent to

$$
j^{4}(g) \sim x^{2} y+\operatorname{sgn}\left(c_{1}\right) y^{4},
$$

provided that $c_{1} \neq 0$. This comes from the fact that all the four-degree monomials of the 4-jet (A9) belong to the Jacobian ideal of $u v^{2}$ except precisely $u^{4}$ and therefore can be removed by a diffeomorphism. From the linear transformation Eq. (A6), we have that

$$
c_{1}=\gamma^{4}\left[b_{1}+b_{2} t_{2}+b_{3} t_{2}^{2}+b_{4} t_{2}^{3}+b_{5} t_{2}^{4}\right]
$$

with $\gamma=1 /\left[a_{4}\left(t_{2}-t_{1}\right)\right]$. Therefore, the sign of $c_{1}$ is given by the factor inside the bracket. Substituting for $t_{2}, c_{1}=\gamma^{4} x_{0}^{-4}$ and is always positive, for any of the three factors given by $\Sigma_{1}=0$, that is $y_{0}=1 / 2, x_{0}=1 / 2$ or $z_{0}=1 / 2$. Finally,

$$
\operatorname{cod}\left(j^{4}(g)\right)=\operatorname{cod}\left(x^{2} y+y^{4}\right)=4 .
$$


$\left(D_{2}\right)$ if $a_{4}=0, a_{1} \neq 0$ (that is, $2 y_{0}=1-x_{0}, 2 x_{0} \neq 1$ $\left.-y_{0}\right)$ and $\Sigma_{1}=0$, then only one point $\left(x_{0}, y_{0}\right)$ needs to be studied, $x_{0}=1 / 2$ and $y_{0}=1 / 4$. The corresponding auxiliary cubic equation is now

$$
0=t^{3}+\frac{a_{2}}{a_{1}} t^{2}+\frac{a_{3}}{a_{1}} t
$$

The roots of this last equation are $t_{1}=0$ and $t_{2}=-2$. With the linear transformation

$$
\psi^{-1} \equiv u=x+2 y, \quad v=2 x
$$

applied to the 4-jet we obtain that

$$
\begin{aligned}
j^{4}(g) \psi(u, v)= & v u^{2}+d_{1} u^{4}+d_{2} u^{3} v+d_{3} u^{2} v^{2}+d_{4} u v^{3} \\
& +d_{5} v^{4}
\end{aligned}
$$

Here the sign of $d_{5}$ is positive and therefore the 4-jet is again equivalent to the canonical germ $x^{2} y+y^{4}$, its codimension being again equal to 4 .

\section{APPENDIX B}

As has been mentioned above we need to show that $h$ is three transversal (similar calculations are needed in order to show that for the hypothesis $\Sigma_{1}=0, h$ is four transversal). One of the main theorems about transversality establishes that this property is fulfilled when

$$
\langle x, y\rangle=\Delta(g)+V_{h}+\langle x, y\rangle^{3+1} \text {. }
$$

The vector space $V_{h}$ is given by Eq. (19) and the ideal of Jacobi of $g$ by

$$
\begin{aligned}
\Delta(g)= & \left\langle D_{x} g(x, y), D_{y} g(x, y)\right\rangle \\
= & \left\langle\frac{1}{2} x^{2} M+x y z_{0}^{-2}+\frac{1}{2} y^{2} z_{0}^{-2}+\frac{1}{3} x^{3}\left(x_{0}^{-3}+z_{0}^{-3}\right)\right. \\
& +x^{2} y z_{0}^{-3}+x y^{2} z_{0}^{-3}+\frac{1}{3} y^{3} z_{0}^{-3}+r(x, y), \frac{1}{2} x^{2} z_{0}^{-2} \\
& +x y z_{0}^{-2}+\frac{1}{2} y^{2} N+\frac{1}{3} x^{3} z_{0}^{-3}+x^{2} y z_{0}^{-3}+x y^{2} z_{0}^{-3} \\
& \left.+\frac{1}{3} y^{3}\left(y_{0}^{-3}+z_{0}^{-3}\right)+s(x, y)\right\rangle
\end{aligned}
$$

with the definitions $M=-x_{0}^{-2}+z_{0}^{-2}$ and $N=-y_{0}^{-2}+z_{0}^{-2}$, and with $r(x, y), s(x, y) \in\langle x, y\rangle^{4}$ after Taylor expansions of $D_{x} g$ and $D_{y} g$ around the point $(0,0)$ have been performed, the first terms being ignored according to the hypothesis $\Sigma_{0}$.

In Eq. (B2), the ideal of Jacobi of $g$ contains monomials of degree greater than 2 . We are going to show that monomials of degree equal to 3 belong to $\Delta\left(j^{3}(g)\right)$. Thus we have that

$$
\begin{aligned}
\lambda_{1} x^{3}+ & \lambda_{2} x^{2} y+\lambda_{3} x y^{2}+\lambda_{4} y^{3} \\
= & (a x+b y)\left(\frac{1}{2} x^{2} M+x y z_{0}^{-2}+\frac{1}{2} y^{2} z_{0}^{-2}\right) \\
& +(c x+d y)\left(\frac{1}{2} x^{2} z_{0}^{-2}+x y z_{0}^{-2}+\frac{1}{2} y^{2} N\right)
\end{aligned}
$$

and the linear system of equations obtained from equating coefficients of the same degree has solutions in $a, b, c$, and $d$ if $\Sigma_{1} \neq 0$.

On the other hand, a straightforward consequence of what we have shown above is that

$$
\Delta(g)+\langle x, y\rangle^{4}=\Delta\left(j^{3}(g)\right)+\langle x, y\rangle^{4}
$$

So finally we can rewrite the transversality condition as

$$
\begin{aligned}
\langle x, y\rangle= & \left\langle\frac{1}{2} x^{2} M+x y z_{0}^{-2}+\frac{1}{2} y^{2} z_{0}^{-2}, \frac{1}{2} x^{2} z_{0}^{-2}+x y z_{0}^{-2}\right. \\
& \left.+\frac{1}{2} y^{2} N\right\rangle+\langle x, y\rangle^{4}+\left\langle-y x-y^{2}+y z_{0}-y_{0} x-y_{0} y\right. \\
& -x^{2}-x y+x z_{0}-x_{0} x-x_{0} y, x y+x y_{0}+x_{0} y,-x, \\
& -y\rangle_{\mathbb{R}} .
\end{aligned}
$$

In other words, we have to find a set of parameters $\nu$ fulfilling

$$
\begin{aligned}
\mu_{1} x+ & \mu_{2} y+\mu_{3} x^{2}+\mu_{4} y^{2}+\mu_{5} x y \\
= & \nu_{1}\left(-y x-y^{2}+y z_{0}-y_{0} x-y_{0} y\right) \\
& +\nu_{2}\left(-x^{2}-x y+x z_{0}-x_{0} x-x_{0} y\right) \\
& +\nu_{3}\left(x y+x y_{0}+x_{0} y\right)+\nu_{4}(-x)+\nu_{5}(-y) \\
& +\nu_{6}\left[\frac{1}{2} x^{2} M+x y \alpha_{0}^{-2}+\frac{1}{2} y^{2} \alpha_{0}^{-2}\right] \\
& +\nu_{7}\left[\frac{1}{2} x^{2} \alpha_{0}^{-2}+x y \alpha_{0}^{-2}+\frac{1}{2} y^{2} N\right]
\end{aligned}
$$

Again by equating coefficients of the same degree we obtain a set of equations between the family of known $\mu$ and unknown $\nu$ parameters. The corresponding system of equations has a matrix of rank equal to 5 and the transversality condition is fulfilled.

For the four-transversal case, the procedure is entirely similar. Only the following observation needs to be made. Monomials of degree 1 and 2 are included in the vector space of transversality and monomials of degree 5 and higher are considered in the term $\langle x, y\rangle^{5}$. So the equivalent to Eq. (B6) has to take into account monomials of degree 3 and 4. This leads to a system of nine equations and ten unknown parameters. The rank of that system is 9 . 
*Also at Laboratorio de Astrofísica Espacial y Física Fundamental, Apartado 50727, 28080 Madrid, Spain.

${ }^{1}$ J. Gaite, J. Margalef-Roig, and S. Miret-Artés, Phys. Rev. B 57, 13527 (1998).

${ }^{2}$ L.D. Landau and E.M. Lifshitz, Statistical Physics (Pergamon, London, 1958).

${ }^{3}$ R. Thom, Stabilité Structurelle et Morphogénese (Benjamin, New York, 1972).

${ }^{4}$ T. Poston and I.N. Stewart, Catastrophe Theory and Its Applications (Pitman, London, 1978); R. Gilmore, Catastrophe Theory for Scientists and Engineers (Dover, New York, 1981).

${ }^{5}$ L.S. Schulman and M. Revzen, Collect. Phenom. 1, 43 (1972); K. Okada, Catastrophe Theory and Phase Transitions (Scitec, Zug, Switzerland, 1993).

${ }^{6}$ J.A. Schouten, A. ten Seldam, and N.J. Trappeniers, Physica (Amsterdam) 73, 556 (1974).

${ }^{7}$ R.B. Griffiths, Phys. Rev. B 12, 345 (1975).

${ }^{8}$ S. Krinsky and D. Mukamel, Phys. Rev. B 11, 399 (1975).

${ }^{9}$ D. Furman, S. Dattagupta, and R.B. Griffiths, Phys. Rev. B 15, 441 (1977).

${ }^{10}$ P.H.E. Meijer and M. Napiórkowski, J. Chem. Phys. 86, 5771 (1987).
${ }^{11}$ L.S. Schulman, Physica A 89, 597 (1977).

${ }^{12}$ J. Gaite, J. Phys. A 25, 3051 (1992), and references therein.

${ }^{13}$ J.S. Rowlinson, Liquids and Liquid Mixtures (Butterworths, London, 1971).

${ }^{14}$ M.A. McHugh and V.J. Krukonis, Supercritical Extraction (Butterworths, London, 1985).

${ }^{15}$ E.C. Zeeman, Catastrophe Theory, Selected Papers 1972-1977 (Addison-Wesley, Reading, MA, 1977).

${ }^{16}$ Th. Bröcker and L. Lander, Differentiable Germs and Catastrophes (Cambridge University Press, Cambridge, 1975).

${ }^{17}$ A.E.R. Woodcock and T. Poston, A Geometrical Study of Elementary Catastrophes, Lecture Notes in Mathematics Vol. 373 (Springer-Verlag, Berlin, 1974).

${ }^{18}$ F.Y. Wu, Rev. Mod. Phys. 54, 235 (1982).

${ }^{19}$ G. Golner, Phys. Rev. B 8, 3419 (1973).

${ }^{20}$ R.K.P. Zia and D. Wallace, J. Phys. A 10, 1495 (1975).

${ }^{21}$ D.M. Burley, Closed Form Approximations for Lattice Systems, in Phase Transitions and Critical Phenomena, Vol. 2, edited by C. Domb and M.S. Green (Academic, London, 1972).

${ }^{22}$ V.I. Arnold, S.M. Gusein-Zade, and A.N. Varchenko, Singularities of Differentiable Maps (Birkäuser, Basel, 1985). 